\title{
Decentralised reliable guaranteed cost control of uncertain systems: an LMI design
}

\author{
G. Pujol, J. Rodellar, J.M. Rossell and F. Pozo
}

\begin{abstract}
The problem of designing a decentralised control scheme for a class of linear large scale interconnected systems with norm-bounded time-varying parameter uncertainties under a class of control failures is addressed. These failures are described by a model that considers possible outages or partial failures in every single actuator of each decentralised controller. The control design is performed through two steps. First, a decentralised reliable guaranteed cost control set is derived and, second, a feasible linear matrix inequalities procedure is presented for the effective construction of the control set. A numerical example illustrates the efficiency of the proposed control scheme.
\end{abstract}

\section{Introduction}

Three important issues arise when dealing with the control of interconnected systems. One concerns the practical limitations in the number and the structure of the feedback loops, which motivates decentralised control schemes. Another aspect concerns the presence of uncertainties both in the subsystems and in the interconnections. A third issue is the reliability of the control systems against component failures. Failures are often encountered in real engineering systems and they may be a source of instability. This is of particular importance in interconnected systems, as failures may appear independently in each subsystem or actuator channel in the form of total outage or partial degradation.

Two main directions have been proposed in the literature for the design of reliable controllers. One uses multiple redundant controls $[1,2]$. The other direction aims to design controls without redundancy by ensuring stability and some degree of performance for specified classes of admissible failures of particular control components [3]. Seeking for stability and performance, classical quadratic optimal control has been used for reliable design [4-6]. In order to address robustness issues, reliable optimal controllers have been designed by using $H_{2} / H_{\infty}$ tools $[7,8]$, integral constraints [9] and the so called guaranteed cost control (GCC).

The GCC approach was proposed as a 'natural' extension of the classical LQ control for linear systems with parametric uncertainties. The conceptual objective is to design a feedback controller such that, for all admissible uncertainties, the closed-loop is asymptotically stable and an upper bound of the quadratic cost is minimised [10-12]. Recently, the potential of linear matrix inequalities (LMIs) has been exploited in the context of GCC. The main issue is to obtain a controller through the solution of an LMI optimisation problem [12-14]. This allows a computationally feasible solution of GCC problems. The GCC

\footnotetext{
(C) The Institution of Engineering and Technology 2007

doi:10.1049/iet-cta:20050364

Paper first received 18th May 2005 and in revised form 2nd June 2006

The authors are with the Department of Applied Mathematics III, Technical University of Catalonia, Comte Urgell 187, Barcelona 08036, Spain

E-mail: gisela.pujol@upc.edu
}

approach has been mostly used for centralised control settings, but it has also been considered for decentralised control of interconnected systems [15-17]. Reliability issues have not been addressed in these references, where a perfect functioning of the controllers is assumed.

When dealing with reliable control design, the GCC approach seeks to ensure robust stability and to retain a degree of performance in the presence of a class of failures. A centralised reliable GCC has been designed by Yang et al. [18] for a class of uncertain nonlinear systems without subsystem interconnections. A condition for decentralised and quadratic stabilisability has been given and used to provide a solution of $H_{2}$-norm optimisation problem [7]. A reliable state feedback controller is synthesised, within the framework of LMI, for delayed systems with integral quadratic constraints [9]. In these papers, system interconnections are not considered. The classes of admissible failures have been usually modelled as outages [7, 9]. This model considers the control set partitioned into one subset with the actuators whose failures are admissible in the control design and a complementary subset with the actuators that are assumed to keep a normal operation [19]. A more general failure scheme is considered [18], where partial failures are also included.

The contribution of this paper is built by combining concepts and tools from the settings considered earlier. This paper develops a method for the design of decentralised reliable GCC systems, using state feedback, for a class of interconnected systems with norm-bounded parametric uncertainties. The class of uncertainties is rather general in the context of robust control $[17,20]$. The system is assumed to operate under failures described by extending the model presented by Yang et al. [18] for the decentralised setting considered here, which allows independent outage or partial degradation in any single actuator of every decentralised controller. This defines a rather general scenario for the design of a decentralised control scheme for a class of systems simultaneously under uncertainties and failures.

The control design is performed through two steps. First, a sufficient condition for the existence of a decentralised reliable GCC set is derived. Then, a feasible LMI procedure is developed for the construction of the control set, allowing its effective implementation. A numerical example shows the efficiency of the controller. 
To the authors knowledge, there are no available results in the literature coping jointly with reliable and robust decentralised control for uncertain interconnected linear systems with guarantee of stability and quadratic performance.

\section{Problem statement}

Consider a class of system decomposed into $N$ interconnected subsystems $S_{i}$ described by the following equations

$$
\dot{\boldsymbol{x}}_{i}(t)=\Delta \boldsymbol{A}_{i}(t) \boldsymbol{x}_{i}(t)+\Delta \boldsymbol{B}_{i}(t) \boldsymbol{u}_{i}(t)+\sum_{j \neq i, j=1}^{N} \boldsymbol{G}_{i j} \boldsymbol{g}_{i j}\left(t, \boldsymbol{x}_{j}\right)
$$

with initial conditions $\boldsymbol{x}_{i}(0)=\boldsymbol{x}_{i 0}$ for $i=1, \ldots, N$. We define $\boldsymbol{x}_{i} \in \mathbb{R}^{n_{i}}$ and $\boldsymbol{u}_{i} \in \mathbb{R}^{s_{i}}$ as the state vector and the control vector of the $i$ th subsystem, respectively, $\Delta \boldsymbol{A}_{i} \in \mathbb{R}^{n_{i} \times n_{i}}$ as state matrix, $\Delta \boldsymbol{B}_{i} \in \mathbb{R}^{n_{i} \times s_{i}}$ as control matrix, $\boldsymbol{g}_{i j}\left(t, \boldsymbol{x}_{j}\right) \in \mathbb{R}^{l_{i}}$ as unknown interconnection vector function and $\boldsymbol{G}_{i j} \in \mathbb{R}^{n_{i} \times l_{i}}$ as constant interconnection matrix. It is assumed that the unknown vectors $\boldsymbol{g}_{i j}\left(t, \boldsymbol{x}_{j}\right)$ are continuous and sufficiently smooth in $\boldsymbol{x}_{j}$ and piecewise continuous in $t$.

\subsection{Uncertainties assumptions}

The parameter uncertainties considered verify the so-called matching-conditions [21] and are assumed to be of the following form

$$
\begin{aligned}
& \Delta \boldsymbol{A}_{i}(t)=\boldsymbol{A}_{i}+\boldsymbol{D}_{i} \boldsymbol{\Delta}_{i}(t) \boldsymbol{E}_{1 i} \\
& \Delta \boldsymbol{B}_{i}(t)=\boldsymbol{B}_{i}+\boldsymbol{D}_{i} \boldsymbol{\Delta}_{i}(t) \boldsymbol{E}_{2 i}
\end{aligned}
$$

where $\boldsymbol{A}_{i} \in \mathbb{R}^{n_{i} \times n_{i}}$ and $\boldsymbol{B}_{i} \in \mathbb{R}^{n_{i} \times s_{i}}$ are known state and control matrices; $\boldsymbol{D}_{i}, \boldsymbol{E}_{1 i}$ and $\boldsymbol{E}_{2 i}$ are known constant matrices of dimensions $n_{i} \times m_{i}, p_{i} \times n_{i}$ and $p_{i} \times s_{i}$, respectively.

Assumption 1: The unknown matrices functions $\boldsymbol{\Delta}_{i}(t) \in$ $\mathbb{R}^{m_{i} \times p_{i}}$ satisfy $\boldsymbol{\Delta}_{i}^{\mathrm{T}}(t) \boldsymbol{\Delta}_{i}(t) \leq \boldsymbol{I}_{p_{i}}$ and $\boldsymbol{\Delta}_{i}(t) \boldsymbol{\Delta}_{i}^{\mathrm{T}}(t) \leq \boldsymbol{I}_{m_{i}}$, for all $t \geq 0$.

Throughout the paper, $\boldsymbol{I}_{p}$ denotes the identity matrix with dimension $p$.

\subsection{Interconnections assumptions}

Assumption 2: There exist known constant matrices $\boldsymbol{W}_{i j}$ such that, for all $\boldsymbol{x}_{j} \in \mathbb{R}^{n_{j}}$

$$
\left\|\boldsymbol{g}_{i j}\left(t, \boldsymbol{x}_{j}\right)\right\| \leq\left\|\boldsymbol{W}_{i j} \boldsymbol{x}_{j}\right\|
$$

for all $i, j$ and for all $t \geq 0$, where \|\| denotes the Euclidean norm.

Assumption 3: For all $i, \sum_{j=1, j \neq \mathrm{i}}^{N} \boldsymbol{W}_{j i}^{\mathrm{T}} \boldsymbol{W}_{j i}>0$.

These two assumptions involve only the unknown interconnection vector function $\boldsymbol{g}_{i j}$, allowing a priori any constant matrix $\boldsymbol{G}_{i j}$. Assumption 2 allows some linear structure to $\boldsymbol{g}_{i j}$ in terms of the state vector $\boldsymbol{x}_{j}$. Assumption 3 will allow to have a well-posed LMI representation later on. This uncertainty structure can represent parametric uncertainties in a wide range of systems [20].

\subsection{Failure model}

Let $\boldsymbol{u}_{i}^{F} \in \mathbb{R}^{s_{i}}$ denote the vector with the signals from the $s_{i}$ actuators that control the $i$ th subsystem. Here, we consider the following failure model

$$
\boldsymbol{u}_{i}^{F}=\boldsymbol{\Lambda}_{i} \boldsymbol{u}_{i}+\boldsymbol{\phi}_{i}\left(\boldsymbol{u}_{i}\right), \quad i=1, \ldots, N
$$

where $\quad \boldsymbol{\Lambda}_{i}=\operatorname{diag}\left(\lambda_{i 1}, \ldots, \lambda_{i s_{i}}\right) \in \mathbb{R}^{s_{i} \times s_{i}}$ is a diagonal positive definite matrix. The uncertain function $\boldsymbol{\phi}_{i}\left(\boldsymbol{u}_{i}\right)=$ $\left(\phi_{i 1}\left(u_{i 1}\right), \ldots, \phi_{i s_{i}}\left(u_{i s_{i}}\right)\right)$ satisfies, for each $i$,

$$
\boldsymbol{\phi}_{i j}^{2} \leq \gamma_{i j}^{2} u_{i j}^{2}, \quad j=1, \ldots, s_{i}
$$

where $\gamma_{i j} \geq 0$. If (4) holds, then

$$
\left\|\boldsymbol{\phi}_{i}\left(\boldsymbol{u}_{i}\right)\right\|^{2} \leq\left\|\boldsymbol{\Gamma}_{i} \boldsymbol{u}_{i}\right\|^{2}, \quad i=1, \ldots, N
$$

where $\boldsymbol{\Gamma}_{i}=\operatorname{diag}\left(\gamma_{i 1}, \ldots, \gamma_{i s_{i}}\right) \in \mathbb{R}^{s_{i} \times s_{i}}$ is a diagonal positive semidefinite matrix.

The value of $\lambda i j$, for $j=1, \ldots, s_{i}$, represents the percentage of failure in the actuator $j$ controlling the subsystem $S_{i}$. With this scheme, each subsystem actuator can fail independently. If $\lambda_{i j}=1$ and $\gamma_{i j}=0$, it corresponds to the normal case for the $j$ th actuator of the $i$ th subsystem $\left(\boldsymbol{u}_{i j}^{F}=\boldsymbol{u}_{i j}\right)$. When this is true for all $j$, we have that $\boldsymbol{\Lambda}_{i}=\boldsymbol{I}_{s_{i}}$ and $\boldsymbol{\Gamma}_{i}=0$, and it corresponds to the normal case in the $i$ th channel $\left(\boldsymbol{u}_{i}^{F}=\boldsymbol{u}_{i}\right)$. When $\lambda_{i j}=\gamma_{i j}$, (3) and (4) covers the outage case $\left(\boldsymbol{u}_{i j}^{F}=0\right)$ because $\boldsymbol{\phi}_{i j}=-\lambda_{i j} u_{i j}$ satisfy (4). The case $\boldsymbol{\phi}_{i}\left(\boldsymbol{u}_{i}\right)=-\boldsymbol{\Lambda}_{i} \boldsymbol{u}_{i}$ corresponds to the outage of the whole controller of the $i$ th system. Other cases correspond to partial failures or partial degradations of the actuators.

\subsection{Control objective}

Consider the following quadratic cost function associated with the system (1)

$$
J(\boldsymbol{x}, \boldsymbol{u})=\sum_{i=1}^{N} \int_{0}^{\infty}\left(\boldsymbol{x}_{i}^{\mathrm{T}}(t) \boldsymbol{Q}_{i} \boldsymbol{x}_{i}(t)+\boldsymbol{u}_{i}^{\mathrm{T}}(t) \boldsymbol{R}_{i} \boldsymbol{u}_{i}(t)\right) \mathrm{d} t
$$

where $\boldsymbol{Q}_{i} \in \mathbb{R}^{n_{i} \times n_{i}}$ and $\boldsymbol{R}_{i} \in \mathbb{R}^{s_{i} \times s_{i}}$ are given constant symmetric positive definite matrices, for all $i$. The objective of this paper is to design a set of decentralised feedback control laws $\left\{\boldsymbol{u}_{i}(t)=\boldsymbol{K}_{i} \boldsymbol{x}_{i}(t), i=1, \ldots, N\right\}$ for the interconnected system (1) with uncertainties model (2), under Assumptions 1, 2 and 3, in such a way that, in the presence of the failures described by (3) and (4), the following properties are satisfied

1.

$$
\sum_{i=1}^{N}\left(\frac{\mathrm{d}}{\mathrm{d} t} \boldsymbol{x}_{i}^{\mathrm{T}}(t) \boldsymbol{P}_{i} \boldsymbol{x}_{i}(t)+\boldsymbol{x}_{i}^{\mathrm{T}}(t) \boldsymbol{Q}_{i} \boldsymbol{x}_{i}(t)+\boldsymbol{u}_{i}^{F}(t)^{\mathrm{T}} \boldsymbol{R}_{i} \boldsymbol{u}_{i}^{F}(t)\right)<0
$$

This inequality leads to a bound for the cost function (6) in the form $J\left(\boldsymbol{x}, \boldsymbol{u}^{F}\right) \leq \bar{J}$, where $\bar{J}$ is some specified constant.

2. The closed-loop subsystems

$$
\begin{aligned}
\dot{\boldsymbol{x}}_{i}(t) & =\Delta \boldsymbol{A}_{i}(t) \boldsymbol{x}_{i}(t)+\Delta \boldsymbol{B}_{i}(t) \boldsymbol{u}_{i}^{F}(t)+\sum_{j \neq i, j=1}^{N} \boldsymbol{G}_{i j} \boldsymbol{g}_{i j}\left(t, x_{j}\right) \\
\boldsymbol{u}_{i}^{F}(t) & =\boldsymbol{\Lambda}_{i} \boldsymbol{u}_{i}(t)+\boldsymbol{\phi}_{i}\left(\boldsymbol{u}_{i}\right) \\
\boldsymbol{u}_{i}(t) & =\boldsymbol{K}_{i} \boldsymbol{x}_{i}(t)
\end{aligned}
$$

are asymptotically stable for each $i=1, \ldots, N$.

Definition 1: The set of above feedback control laws $\left\{\boldsymbol{u}_{i}(t)\right.$, $i=1, \ldots, N\}$ is said to be a reliable GCC set. 


\subsection{Instrumental tool}

The following known result is used in the control design.

Lemma 1 (Schur complement): Consider a symmetric matrix

$$
\boldsymbol{M}=\left(\begin{array}{ll}
\boldsymbol{M}_{1} & \boldsymbol{M}_{2} \\
\boldsymbol{M}_{2}^{\mathrm{T}} & \boldsymbol{M}_{3}
\end{array}\right)
$$

If $\boldsymbol{M}_{3}$ is invertible, $\boldsymbol{S}=\boldsymbol{M}_{1}-\boldsymbol{M}_{2} \boldsymbol{M}_{3}^{-1} \boldsymbol{M}_{2}^{\mathrm{T}}$ is the Schur complement of $\boldsymbol{M}_{3}$ in $\boldsymbol{M}$. Then

1. $\boldsymbol{M}<0$ if and only if $\boldsymbol{M}_{3}<0$ and $\boldsymbol{S}<0$.

2. If $\boldsymbol{M}_{3}<0$, then $\boldsymbol{M} \leq 0$ if and only if $\boldsymbol{S} \leq 0$.

\section{Main results}

The control design involves two steps. First, a sufficient condition is obtained for the existence of a reliable GCC set, in terms of a non-LMI. Second, an LMI procedure is derived for the effective construction of the control set. The currently known LMI characterisations are potentially conservative in the sense that they use a common Lyapunov function regardless of the parameters values. In order to reduce conservatism, we use Assumption 1 to obtain a parametric LMI that does not depend on the system uncertainties.

\subsection{Sufficient condition of existence}

Theorem 1: Under Assumption 2, consider the system (1) with the uncertainties (2) and the failure model (3) and (4). Assume that there exist symmetric positive definite matrices $\boldsymbol{P}_{i} \in \mathbb{R}^{n_{i} \times n_{i}}$ and matrices $\boldsymbol{K}_{i} \in \mathbb{R}^{n_{i} \times s_{i}}$ such that, for all admissible uncertainties $\boldsymbol{\Delta}_{i}(t)$, the inequality $\boldsymbol{M}_{i}<0$ is satisfied, with

$$
\boldsymbol{M}_{i}=\left(\begin{array}{ccccc}
\boldsymbol{\Pi}_{i} & \boldsymbol{P}_{i} \boldsymbol{G}_{i 1} & \cdots & \boldsymbol{P}_{i} \boldsymbol{G}_{i N} & \boldsymbol{\Psi}_{i} \\
\boldsymbol{G}_{i 1}^{\mathrm{T}} \boldsymbol{P}_{i} & -\boldsymbol{I}_{l_{i}} & \cdots & 0 & 0 \\
\vdots & \vdots & \ddots & \vdots & \vdots \\
\boldsymbol{G}_{i N}^{\mathrm{T}} \boldsymbol{P}_{i} & 0 & \cdots & -\boldsymbol{I}_{l_{i}} & 0 \\
\boldsymbol{\Psi}_{i}^{\mathrm{T}} & 0 & \cdots & 0 & \boldsymbol{R}_{i}-\boldsymbol{I}_{s_{i}}
\end{array}\right)
$$

where $\boldsymbol{M}_{i} \in \mathbb{R}^{\bar{N} \times \bar{N}}, \bar{N}=n_{i}+(N-1) l_{i}+s_{i}$ and

$$
\begin{aligned}
\boldsymbol{\Pi}_{i} & :=\overline{\boldsymbol{A}}_{i}^{\mathrm{T}} \boldsymbol{P}_{i}+\boldsymbol{P}_{i} \overline{\boldsymbol{A}}_{i}+\sum_{j=1, j \neq i} \boldsymbol{W}_{j i}^{\mathrm{T}} \boldsymbol{W}_{j i}+\boldsymbol{K}_{i}^{\mathrm{T}} \boldsymbol{\Gamma}_{i}^{2} \boldsymbol{K}_{i}+\overline{\boldsymbol{R}}_{i} \\
\overline{\boldsymbol{A}}_{i} & :=\Delta \boldsymbol{A}_{i}+\Delta \boldsymbol{B}_{i} \boldsymbol{\Lambda}_{i} \boldsymbol{K}_{i} \\
\overline{\boldsymbol{R}}_{i} & :=\boldsymbol{Q}_{i}+\boldsymbol{K}_{i}^{\mathrm{T}} \boldsymbol{\Lambda}_{i} \boldsymbol{R}_{i} \boldsymbol{\Lambda}_{i} \boldsymbol{K}_{i} \\
\boldsymbol{\Psi}_{i} & :=\boldsymbol{P}_{i}\left(\boldsymbol{B}_{i}+\Delta \boldsymbol{B}_{i}\right)+\boldsymbol{R}_{i} \boldsymbol{K}_{i} \boldsymbol{\Lambda}_{i}
\end{aligned}
$$

Then, the set of control laws $\left\{\boldsymbol{u}_{i}(t)=\boldsymbol{K}_{i} \boldsymbol{x}_{i}(t), i=1, \ldots, N\right\}$ is a reliable GCC set. In this case, the corresponding value of the cost function (6) satisfies

$$
J\left(\boldsymbol{x}, \boldsymbol{u}^{F}\right)<\bar{J}:=\sum_{i=1}^{N} \boldsymbol{x}_{i 0}^{\mathrm{T}} \boldsymbol{P}_{i} \boldsymbol{x}_{i 0}
$$

Proof: Let us consider the state feedback control $\boldsymbol{u}_{i}=\boldsymbol{K}_{i} \boldsymbol{x}_{i}$ and the final actual controls after failures $\boldsymbol{u}_{i}^{F}(3)$. By substituting them into (1), we obtain the closed-loop systems

$$
\dot{\boldsymbol{x}}_{i}=\Delta \boldsymbol{A}_{i} \boldsymbol{x}_{i}+\Delta \boldsymbol{B}_{i}\left(\boldsymbol{\Lambda}_{i} \boldsymbol{K}_{i} \boldsymbol{x}_{i}+\boldsymbol{\phi}_{i}\left(\boldsymbol{K}_{i} \boldsymbol{x}_{i}\right)\right)+\sum_{j=1, j \neq i}^{N} \boldsymbol{G}_{i j} \boldsymbol{g}_{i j}\left(\boldsymbol{x}_{j}\right)
$$

Suppose that there exist symmetric positive definite matrices $\boldsymbol{P}_{i}>0$ such that (9) and (10) hold for all admissible uncertainties. In order to prove the asymptotic stability of the closed-loop systems (11), consider the Lyapunov function candidate

$$
\boldsymbol{V}(\boldsymbol{x}):=\sum_{i=1}^{N} \boldsymbol{x}_{i}^{\mathrm{T}} \boldsymbol{P}_{i} \boldsymbol{x}_{i}
$$

where $\boldsymbol{x}^{\mathrm{T}}=\left(\boldsymbol{x}_{1}^{\mathrm{T}}, \ldots, \boldsymbol{x}_{N}^{\mathrm{T}}\right)$. Note that $\boldsymbol{V}(\boldsymbol{x})>0$ holds as $\boldsymbol{P}_{i}>0$ for all $i=1, \ldots, N$, whenever $\boldsymbol{x} \neq 0$. In contrast, the time derivative satisfies

$$
\begin{aligned}
\frac{\mathrm{d}}{\mathrm{d} t}= & \sum_{i=1}^{N} \boldsymbol{x}_{i}^{\mathrm{T}}\left[\left(\Delta \boldsymbol{A}_{i}+\Delta \boldsymbol{B}_{i} \boldsymbol{\Lambda}_{i} \boldsymbol{K}_{i}\right)^{\mathrm{T}} \boldsymbol{P}_{i}+\boldsymbol{P}_{i}\left(\Delta \boldsymbol{A}_{i}+\Delta \boldsymbol{B}_{i} \boldsymbol{\Lambda}_{i} \boldsymbol{K}_{i}\right)\right] \boldsymbol{x}_{i} \\
& +\sum_{i=1}^{N}\left[\boldsymbol{\phi}_{i}^{\mathrm{T}} \Delta \boldsymbol{B}_{i}^{\mathrm{T}} \boldsymbol{P}_{i} \boldsymbol{x}_{i}+\boldsymbol{x}_{i}^{\mathrm{T}} \boldsymbol{P}_{i} \Delta \boldsymbol{B}_{i} \boldsymbol{\phi}_{i}\right] \\
& +\sum_{i=1}^{N}\left[\left(\sum_{j \neq i} \boldsymbol{G}_{i j} \boldsymbol{g}_{i j}\right)^{\mathrm{T}} \boldsymbol{P}_{i} \boldsymbol{x}_{i}+\boldsymbol{x}_{i}^{\mathrm{T}} \boldsymbol{P}_{i}\left(\sum_{j \neq i} \boldsymbol{G}_{i j} \boldsymbol{g}_{i j}\right)\right]
\end{aligned}
$$

Consider $\boldsymbol{z}_{i}^{\mathrm{T}}=\left(\boldsymbol{x}_{i}^{\mathrm{T}}, \boldsymbol{g}_{i 1}^{\mathrm{T}}, \ldots, \boldsymbol{g}_{i N}^{\mathrm{T}}, \boldsymbol{\phi}_{i}^{\mathrm{T}}\right)^{\mathrm{T}}$. Then, (13) becomes

$$
\frac{\mathrm{d}}{\mathrm{d} t} V=\sum_{i=1}^{N}\left[\boldsymbol{z}_{i}^{\mathrm{T}} \boldsymbol{\Omega}_{i} \boldsymbol{z}_{i}+\sum_{j \neq i} \boldsymbol{g}_{i j}^{\mathrm{T}} \boldsymbol{g}_{i j}+\boldsymbol{\phi}_{i}^{\mathrm{T}} \boldsymbol{\phi}_{i}\right]
$$

where $\boldsymbol{\Omega}_{i}$ is defined by

$$
\boldsymbol{\Omega}_{i}=\left(\begin{array}{ccccc}
\overline{\boldsymbol{A}}_{i}^{\mathrm{T}} \boldsymbol{P}_{i}+P_{i} \overline{\boldsymbol{A}}_{i} & \boldsymbol{P}_{i} \boldsymbol{G}_{i 1} & \cdots & \boldsymbol{P}_{i} \boldsymbol{G}_{i N} & P_{i} \Delta \boldsymbol{B}_{i} \\
\boldsymbol{G}_{i 1}^{\mathrm{T}} \boldsymbol{P}_{i} & -\boldsymbol{I}_{l_{i}} & 0 & \cdots & 0 \\
\vdots & \vdots & \ddots & \vdots & \vdots \\
\boldsymbol{G}_{i N}^{\mathrm{T}} \boldsymbol{P}_{i} & 0 & \cdots & -\boldsymbol{I}_{l_{i}} & 0 \\
\Delta \boldsymbol{B}_{i}^{\mathrm{T}} \boldsymbol{P}_{i} & 0 & \cdots & 0 & -\boldsymbol{I}_{s_{i}}
\end{array}\right)
$$

By Assumption 2

$$
\begin{aligned}
\boldsymbol{g}_{i j}^{\mathrm{T}} \boldsymbol{g}_{i j} & \leq \boldsymbol{x}_{j}^{\mathrm{T}} \boldsymbol{W}_{i j}^{\mathrm{T}} \boldsymbol{W}_{i j} \boldsymbol{x}_{j} \\
\sum_{i=1}^{N} \sum_{j \neq i} \boldsymbol{g}_{i j}^{\mathrm{T}} \boldsymbol{g}_{i j} & \leq \sum_{i=1}^{N} \sum_{j \neq i} \boldsymbol{x}_{j}^{\mathrm{T}} \boldsymbol{W}_{i j}^{\mathrm{T}} \boldsymbol{W}_{i j} \boldsymbol{x}_{j} \\
& =\sum_{i=1}^{N} \boldsymbol{x}_{i}^{\mathrm{T}}\left(\sum_{j \neq i} \boldsymbol{W}_{j i}^{\mathrm{T}} \boldsymbol{W}_{j i}\right) \boldsymbol{x}_{i}
\end{aligned}
$$

Therefore

$$
\sum_{i=1}^{N} \sum_{j \neq i} \boldsymbol{g}_{i j}^{\mathrm{T}} \boldsymbol{g}_{i j}-\sum_{i=1}^{N} \boldsymbol{x}_{i}^{\mathrm{T}} \boldsymbol{W}_{i} \boldsymbol{x}_{i} \leq 0
$$

where $\boldsymbol{W}_{i}:=\sum_{j \neq i} \boldsymbol{W}_{j i}^{\mathrm{T}} \boldsymbol{W}_{j i}$. In contrast, using the failure model inequality (5) with $\boldsymbol{u}_{i}=\boldsymbol{K}_{i} \boldsymbol{x}_{i}$, we have

$$
\boldsymbol{\phi}_{i}^{\mathrm{T}} \boldsymbol{\phi}_{i} \leq \boldsymbol{x}_{i}^{\mathrm{T}} \boldsymbol{K}_{i}^{\mathrm{T}} \boldsymbol{\Gamma}_{i}^{2} \boldsymbol{K}_{i} \boldsymbol{x}_{i}
$$


By using $\sum_{i=1}^{N} \boldsymbol{x}_{i}^{\mathrm{T}} \boldsymbol{W}_{i} \boldsymbol{x}_{i}$ and $\boldsymbol{x}_{i}^{\mathrm{T}} \boldsymbol{K}_{i} \boldsymbol{\Gamma}_{i}^{2} \boldsymbol{K}_{i} \boldsymbol{x}_{i}$ in (14), we obtain

$$
\begin{aligned}
\frac{\mathrm{d}}{\mathrm{d} t}= & \sum_{i=1}^{N} \boldsymbol{z}_{i}^{\mathrm{T}} \overline{\boldsymbol{\Omega}}_{i} \boldsymbol{z}_{i}+\sum_{i=1}^{N} \sum_{j \neq i} \boldsymbol{g}_{i j}^{\mathrm{T}} \boldsymbol{g}_{i j}-\sum_{i=1}^{N} \boldsymbol{x}_{i}^{\mathrm{T}} \boldsymbol{W}_{i} \boldsymbol{x}_{i} \\
& +\sum_{i=1}^{N}\left(\boldsymbol{\phi}_{i}^{\mathrm{T}} \boldsymbol{\phi}_{i}-\boldsymbol{x}_{i}^{\mathrm{T}} \boldsymbol{K}_{i}^{\mathrm{T}} \boldsymbol{\Gamma}_{i}^{2} \boldsymbol{K}_{i} \boldsymbol{x}_{i}\right)
\end{aligned}
$$

where $\overline{\boldsymbol{\Omega}}_{i}$ is defined by

$$
\overline{\boldsymbol{\Omega}}_{i}:=\left(\begin{array}{cccc}
\overline{\boldsymbol{A}}_{i}^{\mathrm{T}} \boldsymbol{P}_{i}+\boldsymbol{P}_{i} \overline{\boldsymbol{A}}_{i}+\boldsymbol{W}_{i}+\boldsymbol{K}_{i}^{\mathrm{T}} \boldsymbol{\Gamma}_{i}^{2} \boldsymbol{K}_{i} & \boldsymbol{P}_{i} \boldsymbol{G}_{i 1} & \ldots \\
\boldsymbol{G}_{i 1}^{\mathrm{T}} \boldsymbol{P}_{i} & & -\boldsymbol{I}_{l_{i}} & 0 \\
\vdots & & \vdots & \ddots \\
\boldsymbol{G}_{i N}^{\mathrm{T}} \boldsymbol{P}_{i} & & 0 & \cdots \\
\Delta \boldsymbol{B}_{i}^{\mathrm{T}} \boldsymbol{P}_{i} & & 0 & \ldots \\
& \ldots & \boldsymbol{P}_{i} \boldsymbol{G}_{i N} & \boldsymbol{P}_{i} \Delta \boldsymbol{B}_{i} \\
& \vdots & \vdots & \vdots \\
& & -\boldsymbol{I}_{l_{i}} & 0 \\
& \ldots & 0 & -\boldsymbol{I}_{s_{i}}
\end{array}\right)
$$

If $\overline{\boldsymbol{\Omega}}_{i}<0$ holds, taking into account (15) and (16), we have $\mathrm{d} / \mathrm{dt} V<0$ and the closed-loop systems (11) are asymptotically stable. The matrix $\overline{\boldsymbol{\Omega}}_{i}$ can be decomposed as $\overline{\boldsymbol{\Omega}}_{i}=\boldsymbol{M}_{i}-\boldsymbol{N}_{i}$, where

$$
\boldsymbol{N}_{i}=\left(\begin{array}{ccccc}
\boldsymbol{Q}_{i}+\boldsymbol{K}_{i}^{\mathrm{T}} \boldsymbol{\Lambda}_{i} \boldsymbol{R}_{i} \boldsymbol{\Lambda}_{i} \boldsymbol{K}_{i} & 0 & \cdots & 0 & \boldsymbol{K}_{i}^{\mathrm{T}} \boldsymbol{\Lambda}_{i} \boldsymbol{R}_{i} \\
0 & & & & 0 \\
\vdots & & \ddots & & \vdots \\
0 & & & & 0 \\
\boldsymbol{R}_{i} \boldsymbol{\Lambda}_{i} \boldsymbol{K}_{i} & 0 & \cdots & 0 & \boldsymbol{R}_{i}
\end{array}\right)
$$

Using Lemma 1 for matrix $\boldsymbol{N}_{i}$, it is easy to see that $\overline{\boldsymbol{\Omega}}_{i}<0$.

Next, we are going to prove the inequality (7) and to obtain a bound for the cost function $J\left(\boldsymbol{x}, \boldsymbol{u}^{F}\right)$. By substituting $\boldsymbol{u}_{i}^{F}=\boldsymbol{\Lambda}_{i} \boldsymbol{K}_{i} \boldsymbol{x}_{i}+\boldsymbol{\phi}_{i}$ into (7), we have

$$
\begin{aligned}
\sum_{i=1}^{N}[ & \frac{\mathrm{d}}{\mathrm{d} t}\left(\boldsymbol{x}_{i}^{\mathrm{T}} \boldsymbol{P}_{i} \boldsymbol{x}_{i}\right)+\boldsymbol{x}_{i}^{\mathrm{T}}\left(\boldsymbol{Q}_{i}+\boldsymbol{K}_{i}^{\mathrm{T}} \boldsymbol{\Lambda}_{i} \boldsymbol{R}_{i} \boldsymbol{\Lambda}_{i} \boldsymbol{K}_{i}\right) \boldsymbol{x}_{i} \\
& \left.+\boldsymbol{\phi}_{i}^{\mathrm{T}} \boldsymbol{R}_{i} \boldsymbol{\phi}_{i}+\boldsymbol{\phi}_{i}^{\mathrm{T}} \boldsymbol{R}_{i} \boldsymbol{\Lambda}_{i} \boldsymbol{K}_{i} \boldsymbol{x}_{i}+\boldsymbol{x}_{i}^{\mathrm{T}} \boldsymbol{K}_{i}^{\mathrm{T}} \boldsymbol{\Lambda}_{i} \boldsymbol{R}_{i} \boldsymbol{\phi}_{i}\right] \\
\leq & \sum_{i=1}^{N} \boldsymbol{z}_{i}^{\mathrm{T}} \boldsymbol{M}_{i} \boldsymbol{z}_{i}<0
\end{aligned}
$$

Inequality (17) holds by the assumptions given in (9). Furthermore, from (17), the inequality

$$
\sum_{i=1}^{N}\left[\boldsymbol{x}_{i}^{\mathrm{T}} \boldsymbol{Q}_{i} \boldsymbol{x}_{i}+\left(\boldsymbol{u}_{i}^{F}\right)^{\mathrm{T}} \boldsymbol{R}_{i} \boldsymbol{u}_{i}^{F}\right]<-\sum_{i=1}^{N} \frac{\mathrm{d}}{\mathrm{d} t}\left(\boldsymbol{x}_{i}^{\mathrm{T}} \boldsymbol{P}_{i} \boldsymbol{x}_{i}\right)
$$

holds. By integrating both sides from 0 to $\infty$ and using that $\boldsymbol{V}(\boldsymbol{x}(t)) \rightarrow 0$ when $t \rightarrow \infty$ (by asymptotic stability), we have

$$
J\left(\boldsymbol{x}, \boldsymbol{u}^{F}\right)<\bar{J}:=\sum_{i=1}^{N} \boldsymbol{x}_{i}(0)^{\mathrm{T}} \boldsymbol{P}_{i} \boldsymbol{x}_{i}(0)
$$

This concludes the proof of Theorem 1 .

Remark 1: So far, we have designed a reliable GCC assuming the existence of matrices $\boldsymbol{P}_{i}$ and $\boldsymbol{K}_{i}$ satisfying (9). Note that the bound $\bar{J}$ in (18) depends on the failure model, as $\boldsymbol{M}_{i}$ depends on the matrices $\boldsymbol{\Gamma}_{i}$ and $\boldsymbol{\Lambda}_{i}$ in (9).

\subsection{LMI procedure}

We now present the LMI design approach to the construction of the above reliable GCC set. The main objective is to obtain a feasible LMI that does not depend on the uncertainties. In fact, this LMI is a parametric LMI. These parameters are introduced as variables in the numerical resolution, reducing the conservatism in the control design. The following additional assumption is introduced.

Assumption 4: There exist known constants $\delta_{i}>0$ such that

$$
0<\boldsymbol{E}_{2 i}\left(\boldsymbol{I}_{s_{i}}-\boldsymbol{R}_{i}\right)^{-1} \boldsymbol{E}_{2 i}^{\mathrm{T}}<\delta_{i} \boldsymbol{I}_{p_{i}} \quad \text { for } \quad i=1, \ldots, N .
$$

This assumption introduces some restriction on the control weighting matrices $\boldsymbol{R}_{i}$ in the cost function (6) and on the uncertainties $\boldsymbol{E}_{2 i}$ of the control matrices in (2). This means that we loose some freedom in prescribing the control performance to achieve a reliable control.

Theorem 2: Under Assumptions 1, 2, 3 and 4, suppose that there exist constant parameters $\beta_{1 i}>0, \beta_{2 i}>0, \beta_{3 i}>0$, $\delta_{i}>0$, symmetric positive definite matrices $\boldsymbol{X}_{i} \in \mathbb{R}^{n_{i} \times n_{i}}$ and matrices $\boldsymbol{Y}_{i} \in \mathbb{R}^{s_{i} \times n_{i}}$ such that the LMI

$$
\left(\begin{array}{ll}
\boldsymbol{H}_{i 1} & \boldsymbol{H}_{i 2} \\
\boldsymbol{H}_{i 2}^{\mathrm{T}} & \boldsymbol{H}_{i 3}
\end{array}\right)<0
$$

is feasible for each $i=1, \ldots, N$, where

$$
\begin{aligned}
\boldsymbol{H}_{i 1}= & \boldsymbol{A}_{i} \boldsymbol{X}_{i}+\boldsymbol{X}_{i} \boldsymbol{A}_{i}^{\mathrm{T}}+\boldsymbol{B}_{i}\left(\boldsymbol{I}_{s_{i}}-\boldsymbol{R}_{i}\right)^{-1} \boldsymbol{\Lambda}_{i} \boldsymbol{Y}_{i} \\
& +\boldsymbol{Y}_{i}^{\mathrm{T}} \boldsymbol{\Lambda}_{i} \hat{\boldsymbol{B}}_{i}^{\mathrm{T}}+\sum_{j \neq i} \boldsymbol{G}_{i j} \boldsymbol{G}_{i j}^{\mathrm{T}}+\boldsymbol{B}_{i}\left(\boldsymbol{I}_{s_{i}}-\boldsymbol{R}_{i}\right)^{-1} \boldsymbol{B}_{i}^{\mathrm{T}} \\
& +\left(\delta_{i}+\beta_{1 i}+\beta_{2 i}+\beta_{3 i}\right) \boldsymbol{D}_{i} \boldsymbol{D}_{i}^{\mathrm{T}} \\
\boldsymbol{H}_{i 2}= & \left(\boldsymbol{X}_{i} \boldsymbol{E}_{1 i}^{\mathrm{T}}, \boldsymbol{X}_{i}, \boldsymbol{X}_{i}, \boldsymbol{Y}_{i} \boldsymbol{\Lambda}_{i} \boldsymbol{E}_{2 i}^{\mathrm{T}}, \boldsymbol{Y}_{i}^{\mathrm{T}} \boldsymbol{\Gamma}_{i}, \boldsymbol{Y}_{i}^{\mathrm{T}} \boldsymbol{\Lambda}_{i}, \boldsymbol{Y}_{i}^{\mathrm{T}} \boldsymbol{\Lambda}_{i} \boldsymbol{R}_{i},\right. \\
& \left.\boldsymbol{Y}_{i}^{\mathrm{T}} \boldsymbol{\Lambda}_{i} \boldsymbol{R}_{i}\left(\boldsymbol{I}_{s_{i}}-\boldsymbol{R}_{i}\right)^{-1} \boldsymbol{E}_{2 i}^{\mathrm{T}}, \boldsymbol{B}_{i}\left(\boldsymbol{I}_{s_{i}}-\boldsymbol{R}_{i}\right)^{-1} \boldsymbol{E}_{2 i}^{\mathrm{T}}\right) \\
\boldsymbol{H}_{i 3}= & \operatorname{diag}\left(-\beta_{1 i} \boldsymbol{I}_{n_{i}},-\boldsymbol{W}_{i}^{-1},-\boldsymbol{Q}_{i}^{-1},-\beta_{1 i} \boldsymbol{I}_{n_{i}},-\boldsymbol{I}_{s_{i}},\right. \\
& \left.-\boldsymbol{R}_{i}^{-1}, \boldsymbol{R}_{i}-\boldsymbol{I}_{s_{i}},-\beta_{2 i} \boldsymbol{I}_{s_{i}},-\beta_{3 i} \boldsymbol{I}_{s_{i}}\right)
\end{aligned}
$$

In this case, the decentralised linear state feedback control laws

$$
\boldsymbol{u}_{i}(t)=\boldsymbol{K}_{i} \boldsymbol{x}_{i}(t)=\boldsymbol{Y}_{i} \boldsymbol{X}_{i}^{-1} \boldsymbol{x}_{i}(t), \quad i=1, \ldots, N
$$

are a reliable GCC set and

$$
J\left(\boldsymbol{x}, \boldsymbol{u}^{F}\right)<\bar{J}:=\sum_{i=1}^{N} \boldsymbol{x}_{i 0}^{\mathrm{T}} \boldsymbol{X}_{i}^{-1} \boldsymbol{x}_{i 0}
$$

Proof: The proof basically consists on proving that, under the assumptions of Theorem 2, Theorem 1 is satisfied. Thus, the existence of the reliable GCC set is ensured and, moreover, the matrices $\boldsymbol{P}_{i}$ and $\boldsymbol{K}_{i}$ are characterised by an LMI. First, we decompose $\boldsymbol{M}_{i}$ in (9) in the following form

$$
\boldsymbol{M}_{i}=\left(\begin{array}{ll}
\boldsymbol{M}_{i 1} & \boldsymbol{M}_{i 2} \\
\boldsymbol{M}_{i 2}^{\mathrm{T}} & \boldsymbol{M}_{i 3}
\end{array}\right)
$$

where $\quad \boldsymbol{M}_{i 1}=\boldsymbol{\Pi}_{i}, \quad \boldsymbol{M}_{i 2}=\left(\boldsymbol{P}_{i} \boldsymbol{G}_{i 1}, \ldots, \boldsymbol{P}_{i} \boldsymbol{G}_{i N}, \quad \boldsymbol{\Psi}_{i}\right) \quad$ and $\boldsymbol{M}_{i 3}=\operatorname{diag}\left(-\boldsymbol{I}_{l_{i}}, \ldots,-\boldsymbol{I}_{l_{i}}, \boldsymbol{R}_{i}-\boldsymbol{I}_{s_{i}}\right)$. We have to prove that there exist matrices $\boldsymbol{P}_{i}$ and $\boldsymbol{K}_{i}$ such that $\boldsymbol{M}_{i}<0$ holds. 
By the Schur complement, $\boldsymbol{M}_{i}<0$ holds if and only if

$$
\left\{\begin{array}{l}
\boldsymbol{M}_{i 1}-\boldsymbol{M}_{i 2} \boldsymbol{M}_{i 3}^{-1} \boldsymbol{M}_{i 2}^{\mathrm{T}}<0 \\
\boldsymbol{M}_{i 3}<0
\end{array}\right. \text { and invertible }
$$

The second condition $\boldsymbol{M}_{i 3}<0$ holds if and only if $\left(\boldsymbol{R}_{i}-\boldsymbol{I}_{s_{i}}\right)<0$ and invertible, but this is true by Assumption 4.

The first condition $\boldsymbol{M}_{i 1}-\boldsymbol{M}_{i 2} \boldsymbol{M}_{i 3}^{-1} \boldsymbol{M}_{i 2}^{\mathrm{T}}<0$ can be written in the form

$$
\boldsymbol{\Pi}_{i}+\sum_{j \neq i} \boldsymbol{P}_{i} \boldsymbol{G}_{i j} \boldsymbol{G}_{i j}^{\mathrm{T}} \boldsymbol{P}_{i}-\boldsymbol{\Psi}_{i}\left(\boldsymbol{R}_{i}-\boldsymbol{I}_{s_{i}}\right)^{-1} \boldsymbol{\Psi}_{i}^{\mathrm{T}}<0
$$

In order to prove the inequality (23), two main difficulties have to be faced: (i) the presence of the uncertain matrices $\boldsymbol{\Delta}_{i}(t)$ in $\boldsymbol{\Pi}_{i}$; (ii) the fact that it is nonlinear in $\boldsymbol{P}_{i}$ and $\boldsymbol{K}_{i}$. By using Assumptions 1 and 4 and applying a standard matrix inequality $\left.\left(\boldsymbol{A}^{\mathrm{T}} \boldsymbol{B}+\boldsymbol{B}^{\mathrm{T}} \boldsymbol{A} \leq \boldsymbol{\beta} \boldsymbol{A}^{\mathrm{T}} \boldsymbol{A}+1 / \beta \boldsymbol{B}^{\mathrm{T}} \boldsymbol{B}\right),[22]\right)$, we obtain an inequality that is independent of the uncertainties. By introducing new matrices $\boldsymbol{X}_{i}$ and $\boldsymbol{Y}_{i}$ such that $\boldsymbol{P}_{i}:=\boldsymbol{X}_{i}^{-1}$ and $\boldsymbol{K}_{i}:=\boldsymbol{Y}_{i} \boldsymbol{X}_{i}^{-1}$, it can be proved by using Schur complement that the inequality (23) is equivalent to the linear inequality (19). Consequently, Theorem 2 is proved [23].

The LMI (19) from Theorem 2 is a parametric LMI on $\delta_{i}$, $\beta_{l i}, \beta_{2 i}$ and $\beta_{3 i}$. To reduce the conservatism, they are considered as variables to obtain the reliable GCC for uncertain interconnected systems.

In order to implement the proposed decentralised control set, we have to solve the following problem

\section{$\Sigma_{i 0}$ : Given an initial value $\boldsymbol{x}_{i 0}$, find}

$\left(\boldsymbol{X}_{i}, \boldsymbol{Y}_{i}, \beta_{i 1}, \beta_{i 2}, \beta_{i 3}, \delta_{i}\right)$ such that (19) and (20)

is feasible

The bound of the cost function (22) is dependent on the initial conditions. To remove this dependence, we assume that the initial condition is a zero-mean random variable with $\mathrm{E}\left(\boldsymbol{x}_{i 0}^{\mathrm{T}} \boldsymbol{x}_{i 0}\right)=I d$, where $\mathrm{E}(\cdot)$ denotes the expectation operator. Thus, the cost bound can be written as $\mathrm{E}(J)<\sum_{i=1}^{N} \operatorname{tr}\left(X_{i}^{-1}\right)$, where $\operatorname{tr}(\cdot)$ denotes the trace function. We consider a symmetric matrix $\boldsymbol{Z}_{i}$ such that $\boldsymbol{Z}_{i}>\boldsymbol{X}_{i}^{-1}$. The problem to solve is now, for each $i=1, \ldots, N$

$\Sigma_{i}$ : Minimise $\operatorname{tr}\left(\boldsymbol{Z}_{i}\right)$ over $\left(\boldsymbol{X}_{i}, \boldsymbol{Y}_{i}, \boldsymbol{\beta}_{i 1}, \boldsymbol{\beta}_{i 2}, \boldsymbol{\beta}_{i 3}, \delta_{i}, \boldsymbol{Z}_{i}\right)$

satisfying(19) and (20) and

$$
\left(\begin{array}{cc}
-\boldsymbol{Z}_{i} & \boldsymbol{I}_{n_{i}} \\
\boldsymbol{I}_{n_{i}} & -\boldsymbol{X}_{i}
\end{array}\right)<0
$$

This problem has an additional variable and an additional LMI to solve, but the result is more general than in the case of problem $\Sigma_{i 0}$.

\section{Controller synthesis}

In this section, the steps to solve the reliable GCC problem are discussed. The final goal is to find a feasible solution for the gain matrices and to get a bound for the performance criterion.

Step 1: Define the failure model, the uncertainty model and the interconnection model. It is necessary to know a priori what type of system failures are admitted. It is also necessary to fix the matrices that decompose the system uncertainties (recall that a certain degree of freedom is allowed to choose these matrices). These models have to satisfy Assumptions 1, 2 and 3.

Step 2: Choose the cost function, which must satisfy Assumption 4. In particular, the cost matrices $\boldsymbol{R}_{i}$ have to satisfy the condition $\left(\boldsymbol{I}_{s_{i}}-\boldsymbol{R}_{i}\right)^{-1}>0$. This is easy to verify by a change of variables.

Step 3: Are the initial states $\boldsymbol{x}_{i 0}$ fixed for each $i$ ?

Step 3.1: If yes, solve the problem $\Sigma_{i 0}$ in (24). It is necessary to solve the LMI's (19) and (20) from Theorem 2. If a solution is available, obtain the gain matrix $\boldsymbol{K}_{i}$ for each system $\boldsymbol{S}_{i}, i=1, \ldots, N$ and the cost bound $\bar{J}$. If the LMI's (19) and (20) are not feasible, then revise the uncertainty and failure models. It may be necessary to restrict these models with the aim of having a feasible solution of the problem $\Sigma_{i 0}$.

Step 3.2: If the initial states are not fixed, then solve the problem $\Sigma_{i}$ in $(25)$, with the same restrictions that are in step 3.1.

\section{Design example}

Consider the interconnected uncertain system (1) with three subsystems $S_{1}, S_{2}$ and $S_{3}$. Each subsystem has two states and one control input, with matrices

$$
\begin{aligned}
& S_{1}:\left\{\begin{array}{c}
\boldsymbol{A}_{1}=\left[\begin{array}{cc}
0.1 & 0 \\
0 & 1
\end{array}\right], \boldsymbol{B}_{1}=\left[\begin{array}{l}
2 \\
1
\end{array}\right] \\
\boldsymbol{G}_{12}=\left[\begin{array}{c}
0.01 \\
0
\end{array}\right], \boldsymbol{G}_{13}=\left[\begin{array}{c}
0 \\
0.1
\end{array}\right]
\end{array}\right\} \\
& S_{2}:\left\{\begin{array}{c}
\boldsymbol{A}_{2}=\left[\begin{array}{cc}
0.1 & -1 \\
0 & 1
\end{array}\right], \boldsymbol{B}_{2}=\left[\begin{array}{c}
1 \\
0.5
\end{array}\right] \\
\boldsymbol{G}_{21}=\left[\begin{array}{c}
0.04 \\
0
\end{array}\right], \boldsymbol{G}_{23}=\left[\begin{array}{c}
0 \\
0.1
\end{array}\right]
\end{array}\right\} \\
& S_{3}:\left\{\begin{array}{c}
\boldsymbol{A}_{3}=\left[\begin{array}{cc}
1 & -0.1 \\
1 & 1
\end{array}\right], \boldsymbol{B}_{3}=\left[\begin{array}{l}
1 \\
1
\end{array}\right] \\
\boldsymbol{G}_{31}=\left[\begin{array}{c}
0 \\
0.2
\end{array}\right], \boldsymbol{G}_{32}=\left[\begin{array}{c}
0 \\
0.1
\end{array}\right]
\end{array}\right\}
\end{aligned}
$$

The interconnection vectors $\boldsymbol{g}_{i j}\left(t, \boldsymbol{x}_{j}\right)$ are bounded by $\boldsymbol{W}_{i j}=\operatorname{diag}(0.1,0.1)$ for all $i, j=1,2,3$, while the uncertain matrices in (2) are

$$
\begin{gathered}
\boldsymbol{D}_{i}=\left[\begin{array}{cc}
0.1 & 0 \\
0 & 0
\end{array}\right], \quad \boldsymbol{E}_{1 i}=\left[\begin{array}{ll}
0 & 1
\end{array}\right], \quad \boldsymbol{E}_{2 i}=[0.1] \\
\boldsymbol{\Delta}(t)=\left[\begin{array}{ll}
0.1 \sin (t) & 0.1
\end{array}\right]^{\mathrm{T}}
\end{gathered}
$$

We consider the cost matrices $\boldsymbol{R}_{i}=[0.7]$ and $\boldsymbol{Q}_{i}=$ $\operatorname{diag}(0.01,0.01)$ and the initial states $\boldsymbol{x}_{i 0}=[1,0.5]^{\mathrm{T}}$ for $i=1,2,3$.

In order to demonstrate the effectiveness of the proposed reliable control algorithm, we first consider the nominal control design assuming an 'ideal' scenario in which no actuator failures will occur. Then, by solving the problem $\Sigma_{i 0}$ in (24), with $\boldsymbol{\Lambda}_{i}=1$ and $\boldsymbol{\Gamma}_{i}=0$ for $i=1,2,3$, the following control gain matrices are obtained

$$
\begin{aligned}
& \boldsymbol{K} \boldsymbol{N}_{1}=[2.10,-9.94] \\
& \boldsymbol{K} \boldsymbol{N}_{2}=[2.34,-15.97] \\
& \boldsymbol{K N}_{3}=[10.29,-20.98]
\end{aligned}
$$

The resulting cost bound is $\bar{J}_{\text {ideal }}=7.11$.

Consider now the objective of designing the proposed reliable control scheme for a failure scenario described by 
the following model

$$
\begin{array}{lll}
S_{1}: & u_{1}^{F}=0.5 u_{1}+\phi_{1}, & \phi_{1}^{2} \leq 0.1^{2} u_{1}^{2} \\
S_{2}: & u_{2}^{F}=0.9 u_{2}+\phi_{2}, & \phi_{2}^{2} \leq 0.3^{2} u_{2}^{2} \\
S_{3}: & u_{3}^{F}=0.7 u_{3}+\phi_{3}, & \phi_{3}^{2} \leq 0.2^{2} u_{3}^{2}
\end{array}
$$

This means that the designer allows a failure of the order of $50 \%$ in the actuator of system $S_{1}$ with an error of the order of $10 \%$. The tolerances assumed for the other actuators are interpreted in the same way.

With this aim, we apply the design procedure of Section 4, where the parameters defined in (3) and (4) are: $\Lambda_{1}=0.5$, $\Gamma_{1}=0.1, \Lambda_{2}=0.9, \Gamma_{2}=0.3, \Lambda_{3}=0.7, \Gamma_{3}=0.2$. Solving the problem $\Sigma_{i 0}$ in (24), we obtain the control gain matrices

$$
\begin{aligned}
& \boldsymbol{K R}_{1}=[4.38,-20.62] \\
& \boldsymbol{K R}_{2}=[2.69,-19.38] \\
& \boldsymbol{K R}_{3}=[12.14,-27.68]
\end{aligned}
$$

with the cost bound $\bar{J}_{0}=8.14$.

Three numerical experiments are performed for three operation modes.

1. Ideal mode, where the nominal controllers $\boldsymbol{K} \boldsymbol{N}$ (26) are implemented in an operation without failures.

2. Failure mode, where the nominal controllers $\boldsymbol{K} \boldsymbol{N}$ (26) are implemented in an operation under the failures described in (27).

3. Reliable control failure mode, where the reliable controllers $\boldsymbol{K} \boldsymbol{R}(28)$ are implemented in an operation under the failures described in (27).

Figs. 1 and 2 show the state $x_{11}$ and the control signal $u_{1}$ of system $S_{1}$, respectively, for these three modes. Similar patterns are observed for the states and control signals of systems $S_{2}$ and $S_{3}$, which are not shown here for the sake of brevity.

We observe that the nominal control clearly deteriorates its ideal performance when operating under failures. The state response has a significant overshoot and has a slower response when compared with the ideal case, whereas the control signal requires a significantly larger magnitude. When using the reliable control, we notice

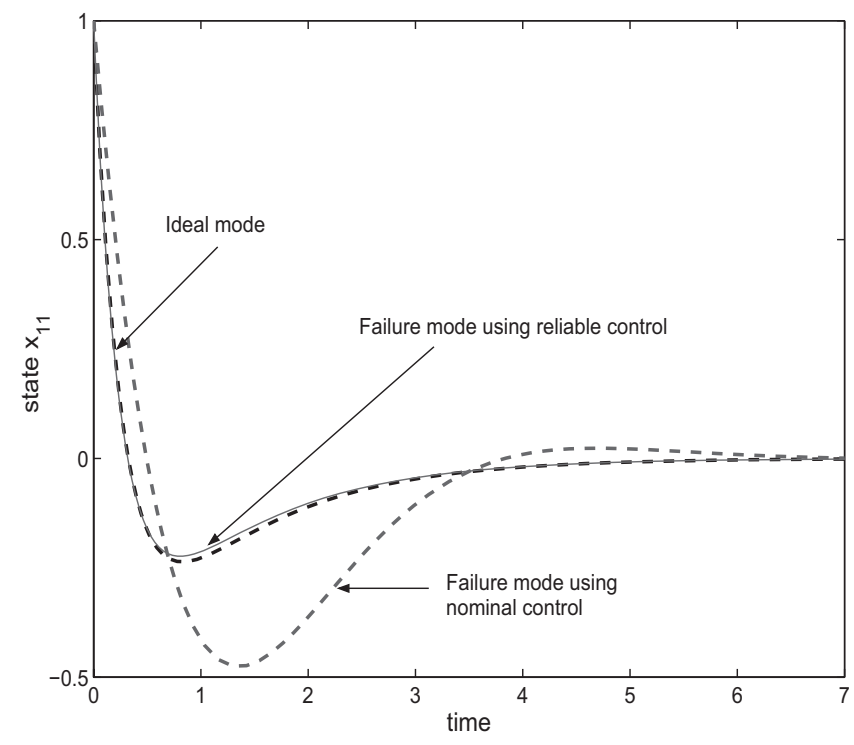

Fig. 1 State $x_{11}$ of system $S_{1}$ for the three operation modes: ideal, failure with nominal control, failure with reliable control

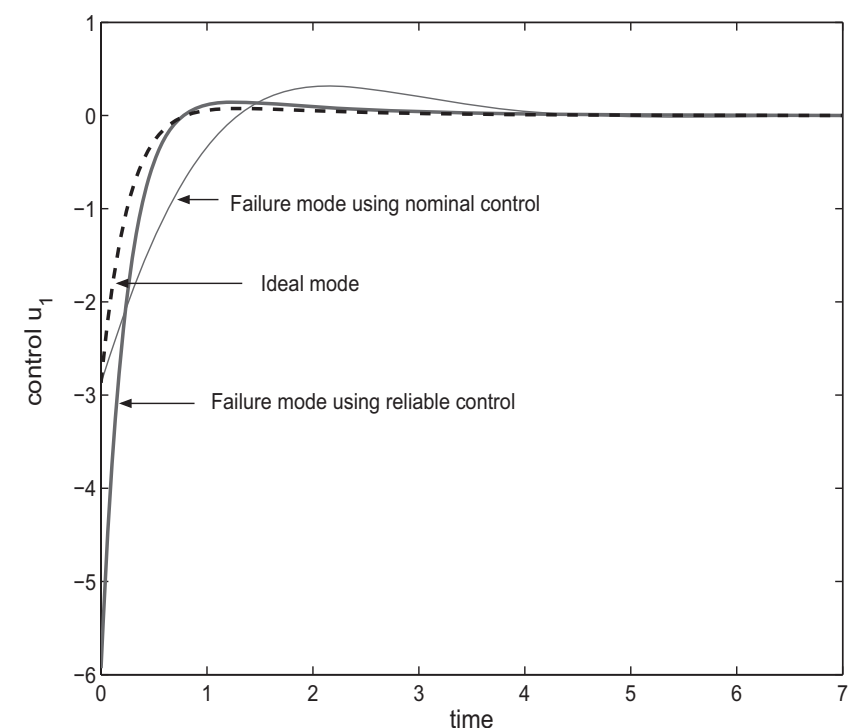

Fig. 2 Control signal $u_{1}$ of system $S_{I}$ for the three operation modes: ideal, failure with nominal control, failure with reliable control

that the state response results drastically improved in spite of working under the same failures, being very close to the one obtained in the ideal case. As Fig. 2 shows, the control effort in this case has a bigger initial magnitude than for the nominal cases, quickly approaching the ideal control signals.

These results illustrate the satisfactory behaviour of the proposed reliable control scheme. According to the design objectives, the control ensures the stability and a quadratic performance criterion under a computed bound for the assumed uncertainties and the admitted failure scenario. Regarding the performance bound, it is interesting to compare both values $\bar{J}_{\text {ideal }}=7.11$ and $\bar{J}_{0}=8.14$ for the nominal and the reliable control, respectively. The bigger value of the bound for the reliable case is understandable, as the control is designed to keep stability and performance in spite of possible failures, so a worse performance is reasonably expected. In the numerical example, this worse performance seems to be related to the bigger initial control magnitude required to keep the state under failures close to the ideal response.

This example has illustrated a control design in which the initial states $x_{i 0}$ are known, which corresponds to the problem $\Sigma_{i 0}$ in (24). If the initial conditions are not prescribed, the problem $\Sigma_{i}$ in (25) must be solved. In this case, the computed bounds result in $\bar{J}_{\text {ideal }}^{\prime}=26.24$ and $\bar{J}_{0}^{\prime}=29.57$, respectively, which are bigger than the values $\bar{J}_{\text {ideal }}=7.11$ and $\bar{J}_{0}=8.14$, respectively. These bigger values are expected because this design covers all possible initial states.

The LMI's (19), (20) and (25) have been solved using MATLAB's LMI Control Toolbox.

\section{Conclusions}

In this paper, a solution of a reliable GCC problem for a class of linear interconnected systems with norm-bounded parameter uncertainties has been presented. Failures are described by a model that considers possible outage or partial failures in every actuator of each decentralised controller. The control design involves two steps. First, a sufficient condition is given for the existence of a decentralised reliable GCC set. Second, this control is effectively 
constructed by means of a feasible LMI optimisation problem. A key point in the control design has been the formulation of an LMI characterisation, which uses assumptions over model uncertainties to remove the parameter dependence of the control characterisation. The design presented in this paper shows that the presence of control failures imposes some restrictions in the definition of the cost function to be bounded. Specifically, some freedom is lost in the selection of the control weighting matrices. Thus, the designer can take some trade-off between control performance and admitted reliability. A numerical example has been included to illustrate the efficiency of the proposed decentralised control approach to ensure stability and performance in the presence of both control failures and parametric uncertainties.

\section{Acknowledgments}

The authors would like to thank the reviewers for their helpful suggestions. This work was funded by CICYTSpain under project DPI2005-08668-C03-01.

\section{References}

1 Siljak, D.D.: 'Reliable control using redundant controllers', IEEE Trans. Autom. Control, 1980, 31, pp. 303-329

2 Lam, J., Yang, G.-H., Zhang, S.-Y., and Wang, J.: 'Reliable control using redundant controllers', IEEE Trans. Autom. Control, 1998, 43, pp. $1588-1593$

3 McFarlane, A.C.J.: 'Complex variable methods for linear multivariable feedback systems' (Taylor and Francis, London, 1980)

4 Veillette, R.J.: 'Reliable linear quadratic state feedback control', Automatica, 1995, 31, pp. 137-143

5 Zhao, Q., and Jiang, J.: 'Reliable state feedback control system design actuator failures', Automatica, 1998, 34, pp. 1267-1272

6 Hsieh, C.S.: 'Reliable control design using a two-stage linear quadratic reliable control', IEE Proc. Control Theory Appl., 2003, 150, (1), pp. 77-82

7 Geromel, J.C., Bernussou, J., and de Oliveira, M.C.: 'H2-norm optimization with constrained dynamic output feedback controllers: decentralized and reliable control', IEEE Trans. Autom. Control, 1999, 44, (7), pp. 1449-1454
8 Yang, G.-H., Wang, J.L., and Soh, Y.C.: 'Reliable $H_{\infty}$ controller design for linear systems', Automatica, 2001, 37, (5), pp. 717-725

9 Cheng, C., and Zhao, Q.: 'Reliable control of uncertain delayed systems with integral quadratic constraints', IEE Proc. Control Theory Appl., 2004, 151, (6), pp. 790-796

10 Petersen, I.R., and McFarlane, D.C.: 'Optimal guaranteed cost control and filtering for uncertain linear systems', IEEE Trans. Autom. Control, 1994, 39, pp. 1971-1977

11 Yang, G.-H., Wang, J.L., and Soh, Y.C.: 'Guaranteed cost control for discrete-time linear systems under controller gain perturbations', Linear Algebr. Appl., 2000, 312, pp. 162-180

12 Costa, E.F., and Oliveira, V.A.: 'On the design of guaranteed cost controllers for a class of uncertain linear systems', Syst. Control Lett., 2001, 46, pp. 17-29

13 Xu, S., Lam, J., Yang, C., and Verriest, E.I.: 'An LMI approach to guaranteed cost control for uncertain linear neutral delay systems', Int. J. Robust Nonliner Control, 2003, 13, pp. 35-53

$14 \mathrm{Yu}, \mathrm{L}$., and Gao, F.: 'Output feedback guaranteed cost control for uncertain discrete-time systems using linear matrix inequalities', J. Optim. Theory Appl., 2002, 113, (3), pp. 621-634

15 Xie, S., Xie, L., Wang, Y., and Guo, G.: 'Decentralized control of multimachine power systems with guaranteed performance', IEE Proc. Control Theory Appl., 2000, 147, (3), pp. 355-365

16 Gao, J., and Huang, B.: 'Delay-dependent robust guaranteed cost control of an uncertain linear system with state and input delay', Int. J. Syst. Sci., 2005, 36, (1), pp. 19-26

17 Mukaidini, H.: 'An LMI approach to decentralized guaranteed cost control for a class of uncertain nonlinear large-scale delay systems', J. Math. Anal. Appl., 2004, 300, pp. 17-29

18 Yang, G.-H., Wang, J.L., and Soh, Y.C.: 'Reliable guaranteed cost control for uncertain nonlinear systems', IEEE Trans. Autom. Control, 2000, 45, (11), pp. 2188-2192

19 Liao, F., Wang, J.L., and Yang, G-H.: 'Reliable robust flight tracking control: an LMI approach', IEEE Trans. Control Syst. Technol., 2002, 10, (1), pp. 76-89

20 Wang, Y., Hill, D.J., and Guo, G.: 'Robust decentralized control for multimachine power systems', IEEE Trans. Circuits Syst., 1998, 45, (3), pp. 271-279

21 Leitmann, G.: 'On one approach to the control of uncertain systems', J. Dyn. Syst., Meas. Control, 1993, 115, pp. 373-380

22 Khargonekar, P., Petersen, I., and Zhou, K.: 'Robust stabilization of uncertain linear systems: quadratic stabilization and $H_{\infty}$ control theory', IEEE Trans. Autom. Control, 1990, 35, (3), pp. 356-361

23 Pujol, G.: 'Contribution on reliable control for uncertain interconnected systems'. $\mathrm{PhD}$ Thesis, Technical University of Catalonia, Spain, 2004 